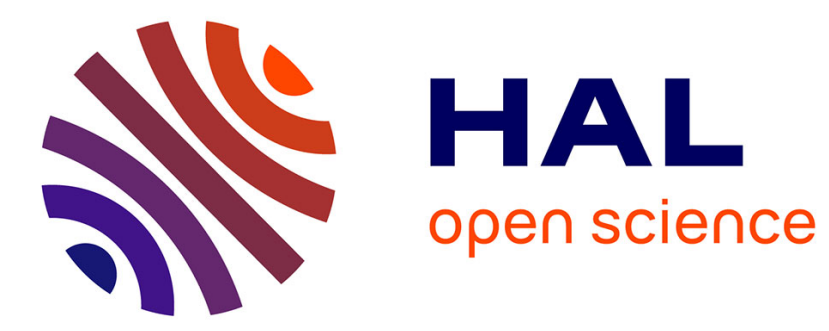

\title{
Power Laws Variance Scaling of Boolean Random Varieties
}

\author{
Dominique Jeulin
}

\section{To cite this version:}

Dominique Jeulin. Power Laws Variance Scaling of Boolean Random Varieties. Methodology and Computing in Applied Probability, 2015, 10.1007/s11009-015-9464-5 . hal-01207456

\section{HAL Id: hal-01207456 \\ https:/ /hal-mines-paristech.archives-ouvertes.fr/hal-01207456}

Submitted on 30 Sep 2015

HAL is a multi-disciplinary open access archive for the deposit and dissemination of scientific research documents, whether they are published or not. The documents may come from teaching and research institutions in France or abroad, or from public or private research centers.
L'archive ouverte pluridisciplinaire HAL, est destinée au dépôt et à la diffusion de documents scientifiques de niveau recherche, publiés ou non, émanant des établissements d'enseignement et de recherche français ou étrangers, des laboratoires publics ou privés. 


\title{
Power laws variance scaling of Boolean random varieties
}

\author{
Dominique Jeulin \\ Centre de Morphologie Mathématique \\ Mines ParisTech \\ PSL Research University \\ 35 rue Saint-Honoré, F77300 Fontainebleau, France \\ email: dominique.jeulin@mines-paristech.fr
}

26-08-2015

\begin{abstract}
Long fibers or stratified media show very long range correlations. These media can be simulated by models of Boolean random varieties and their iteration. They show non standard scaling laws with respect to the volume of domains $K$ for the variance of the local volume fraction: on a large scale, the variance of the local volume fraction decreases according to power laws of the volume of $K$. The exponent $\gamma$ is equal to $\frac{n-k}{n}$ for Boolean varieties with dimension $k$ in the space $\mathbb{R}^{n}: \gamma=\frac{2}{3}$ for Boolean fibers in 3D, and $\gamma=\frac{1}{3}$ for Boolean strata in $3 \mathrm{D}$. When working in $2 \mathrm{D}$, the scaling exponent of Boolean fibers is equal to $\frac{1}{2}$. From the results of numerical simulations, these scaling laws are expected to hold for the prediction of the effective properties of such random media.
\end{abstract}

Keywords: Boolean model, random fiber networks, random strata, Poisson varieties, RVE, integral range, long range correlations, scaling law, numerical homogenization

AMS 2000 Subject Classifications: 60G55, 60D05, 52A22

\section{Introduction}

The scaling of fluctuations of morphological properties like the volume fraction, or of local fields (such as electrostatic or elastic fields) is necessary to define the size of a statistical representative element (RVE). For years a geostatistical approach (Matheron 1971) was used in image analysis for this purpose (Hersant and Jeulin 1976). It was recently extended to the computation of effective properties by numerical homogenization (Cailletaud et al. 1994; Jeulin 2005; Kanit et al. 2003). The calculation of the scaling of 
the variance makes use of the integral of the centred covariance, namely the integral range. In some situations with very long range correlations, it turns out that the integral range is infinite, and new scaling laws of the variance can occur (Lantuejoul 1991). In this paper, we study such models of random sets, the Boolean models built on the Poisson varieties, generating for instance random fiber networks or random strata made of dilated planes in the three-dimensional space.

After a reminder on Poisson varieties (Matheron 1975), of Boolean random varieties (Jeulin 1991; Jeulin 1991) and on the statistical definition of the RVE for the volume fraction, we give theoretical results on the scaling of the variance of the Boolean random varieties and of their iterated version.

\section{Poisson varieties}

\subsection{Construction and properties of the linear Poisson vari- eties model in $\mathbb{R}^{n}$}

A geometrical introduction of the Poisson linear varieties is as follows (Matheron 1975), : a Poisson point process $\left\{x_{i}(\omega)\right\}$, with intensity $\theta_{k}(d \omega)$ is considered on the varieties of dimension $(n-k)$ containing the origin $O$, and with orientation $\omega$. On every point $x_{i}(\omega)$ is located a variety with dimension $k, V_{k}(\omega)_{x_{i}}$, orthogonal to the direction $\omega$. By construction, we have $V_{k}=\cup_{x_{i}(\omega)} V_{k}(\omega)_{x_{i}}$. For instance in $\mathbb{R}^{3}$ can be built a network of Poisson hyperplanes $\Pi_{\alpha}$ (orthogonal to the lines $D_{\omega}$ containing the origin) or a network of Poisson lines in every plane $\Pi_{\omega}$ containing the origin (figure 1).

Definition 1 In $\mathbb{R}^{n}$, n Poisson linear varieties of dimension $k(k=0,1, \ldots, n-$ 1) $V_{k}$, can be built: for $k=0$ is obtained the Poisson point process, and for $k=n-1$ are obtained the Poisson hyperplanes. For $k \geq 1$, a network of Poisson linear varieties of dimension $k$ can be considered as a Poisson point process in the space $S_{k} \times \mathbb{R}^{n-k}$, with intensity $\theta_{k}(d \omega) \mu_{n-k}(d x) ; \theta_{k}$ is a positive Radon measure for the set of subspaces of dimension $k, S_{k}$, and $\mu_{n-k}$ is the Lebesgue measure of $\mathbb{R}^{n-k}$.

If $\theta_{k}(d \omega)$ is any Radon measure, the obtained varieties are anisotropic. When $\theta_{k}(d \omega)=\theta_{k} d \omega$, the varieties are isotropic. If the Lebesgue measure $\mu_{n-k}(d x)$ is replaced by a measure $\theta_{n-k}(d x)$, we obtain non stationary random varieties.

The probabilistic properties of the Poisson varieties are easily derived from their definition as a Poisson point process.

Theorem 2 The number of varieties of dimension $k$ hit by a compact set $K$ is a Poisson variable, with parameter $\theta(K)$ :

$$
\theta(K)=\int \theta_{k}(d \omega) \int_{K(\omega)} \theta_{n-k}(d x)=\int \theta_{k}(d \omega) \theta_{n-k}(K(\omega))
$$


where $K(\omega)$ is the orthogonal projection of $K$ on the orthogonal space to $V_{k}(\omega), V_{k^{\perp}}(\omega)$. For the stationary case,

$$
\theta(K)=\int \theta_{k}(d \omega) \mu_{n-k}(K(\omega))
$$

The Choquet capacity $T(K)=P\left\{K \cap V_{k} \neq \varnothing\right\}$ of the varieties of dimension $k$ is given by

$$
T(K)=1-\exp \left(-\int \theta_{k}(d \omega) \int_{K(\omega)} \theta_{n-k}(d x)\right)
$$

In the stationary case, the Choquet capacity is

$$
T(K)=1-\exp \left(-\int \theta_{k}(d \omega) \mu_{n-k}(K(\omega))\right)
$$

Proof. By construction, the random varieties $V_{k}(\omega)$ induce by intersection on every orthogonal variety of dimension $n-k, V_{k^{\perp}}(\omega)$, a Poisson point process with dimension $n-k$ and with intensity $\theta_{k}(d \omega) \theta_{n-k}(d x)$. Therefore, the contribution of the direction $\omega$ to $N(K)$, is the Poisson variable $N(K, \omega)$ with intensity $\theta_{n-k}(K(\omega))$. Since the contributions of the various directions are independent, Eq. (1) results immediately.

Proposition 3 We consider now the isotropic ( $\theta_{k}$ being constant) and stationary case, and a convex set $K$. Due to the symmetry of the isotropic version, we can consider $\theta_{k}(d \omega)=\theta_{k} d \omega$ as defined on the half unit sphere (in $\mathbb{R}^{k+1}$ ) of the directions of the varieties $V_{k}(\omega)$. The number of varieties of dimension $k$ hit by a compact set $K$ is a Poisson variable, with parameter $\theta(K)$ given by:

$$
\theta(K)=\theta_{k} \int \mu_{n-k}(K(\omega)) d \omega=\theta_{k} \frac{b_{n-k} b_{k+1}}{b_{n}} \frac{k+1}{2} W_{k}(K)
$$

where $b_{k}$ is the volume of the unit ball in $\mathbb{R}^{k}\left(b_{k}=\frac{\pi^{k / 2}}{\Gamma\left(1+\frac{k}{2}\right)}\right)\left(b_{1}=2, b_{2}=\right.$ $\left.\pi, b_{3}=\frac{4}{3} \pi\right)$, and $W_{k}(K)$ is the Minkowski's functional of $K$, homogeneous and of degree $n-k$ (Matheron 1975).

The following examples are useful for applications:

- When $k=n-1$, the varieties are Poisson planes in $\mathbb{R}^{n}$; in that case, $\theta(K)=\theta_{n-1} n W_{n-1}(K)=\theta_{n-1} \mathcal{A}(K)$, where $\mathcal{A}(K)$ is the norm of $K$ (average projected length over orientations). 
- In the plane $\mathbb{R}^{2}$ are obtained the Poisson lines, with $\theta(K)=\theta L(K)$, $L$ being the perimeter.

- In the three-dimensional space are obtained Poisson lines for $k=1$ and Poisson planes for $k=2$. For Poisson lines, $\theta(K)=\frac{\pi}{4} \theta S(K)$ and for Poisson planes, $\theta(K)=\theta M(K)$, where $S$ and $M$ are the surface area and the integral of the mean curvature.

\section{Boolean random varieties}

Boolean random sets can be built, starting from Poisson varieties and a random primary grain (Jeulin 1991, 1991).

Definition 4 A Boolean model with primary grain $A^{\prime}$ is built on Poisson linear varieties in two steps: i) we start from a network $V_{k}$; ii) every variety $V_{k \alpha}$ is dilated by an independent realization of the primary grain $A^{\prime}$. The Boolean RACS A is given by

$$
A=\cup_{\alpha} V_{k \alpha} \oplus A^{\prime}
$$

By construction, this model induces on every variety $V_{k^{\perp}}(\omega)$ orthogonal to $V_{k}(\omega)$ a standard Boolean model with dimension $n-k$, with random primary grain $A^{\prime}(\omega)$ and with intensity $\theta(\omega) d \omega$. The Choquet capacity of this model immediately follows, after averaging over the directions $\omega$; it can also be deduced from Eq. (4), after replacing $K$ by $A^{\prime} \oplus \check{K}$ and averaging.

Theorem 5 The Choquet capacity of the Boolean model built on Poisson linear varieties of dimension $k$ is given by

$$
T(K)=1-\exp \left(-\int \theta_{k}(d \omega) \bar{\mu}_{n-k}\left(A^{\prime}(\omega) \oplus \check{K}(\omega)\right)\right)
$$

For isotropic varieties, the Choquet capacity of Boolean varieties is given by

$$
T(K)=1-\exp \left(-\theta_{k} \frac{b_{n-k} b_{k+1}}{b_{n}} \frac{k+1}{2} \bar{W}_{k}\left(A^{\prime} \oplus \check{K}\right)\right)
$$

Particular cases of Eq. (6) are obtained when $K=\{x\}$ (giving the probability $q=P\left\{x \in A^{c}\right\}=\exp \left(-\int \theta_{k}(d \omega) \mu_{n-k}\left(A^{\prime}(\omega)\right)\right)$ and when $K=\{x, x+h\}$, giving the covariance of $A^{c}, Q(h)$ :

$$
Q(h)=q^{2} \exp \left(\int \theta_{k}(d \omega) K_{n-k}(\omega, \vec{h} \cdot \vec{u}(\omega))\right)
$$


where $K_{n-k}(\omega, h)=\bar{\mu}_{n-k}\left(A^{\prime}(\omega) \cap A_{-h}^{\prime}(\omega)\right)$ and $\vec{u}(\omega)$ is the unit vector with the direction $\omega$. For a compact primary grain $A^{\prime}$, there exists for any $h$ an angular sector where $K_{n-k}(\omega, h) \neq 0$, so that the covariance generally does not reach its sill, at least in the isotropic case, and the integral range, defined in section (4.1), is infinite. We consider now some examples.

\subsection{Fibers in 2D}

In the plane can be built a Boolean model on Poisson lines. For an isotropic lines network (figure 1 ), and if $A^{\prime} \oplus \check{K}$ is a convex set, we have, from equation (7):

$$
T(K)=1-\exp \left(-\theta \bar{L}\left(A^{\prime} \oplus \check{K}\right)\right)
$$

If $A^{\prime} \oplus \check{K}$ is not a convex set, the integral of projected lengths over a line with the orientation varying between 0 and $\pi$ must be taken. If $A^{\prime}$ and $K$ are convex sets, we have $\bar{L}\left(A^{\prime} \oplus \check{K}\right)=\bar{L}\left(A^{\prime}\right)+L(K)$. Consider now the isotropic case. Using for $A^{\prime}$ a random disc with a random radius $R$ (with expectation $\bar{R}$ ) and for $K$ a disc with radius $r$, equation 9 becomes:

$$
\begin{aligned}
& T(r)=1-\exp (-2 \pi \theta(\bar{R}+r)) \\
& T(0)=P\{x \in A\}=1-\exp (-2 \pi \theta \bar{R})
\end{aligned}
$$

which can be used to estimate $\theta$ and $\bar{R}$, and to validate the model.

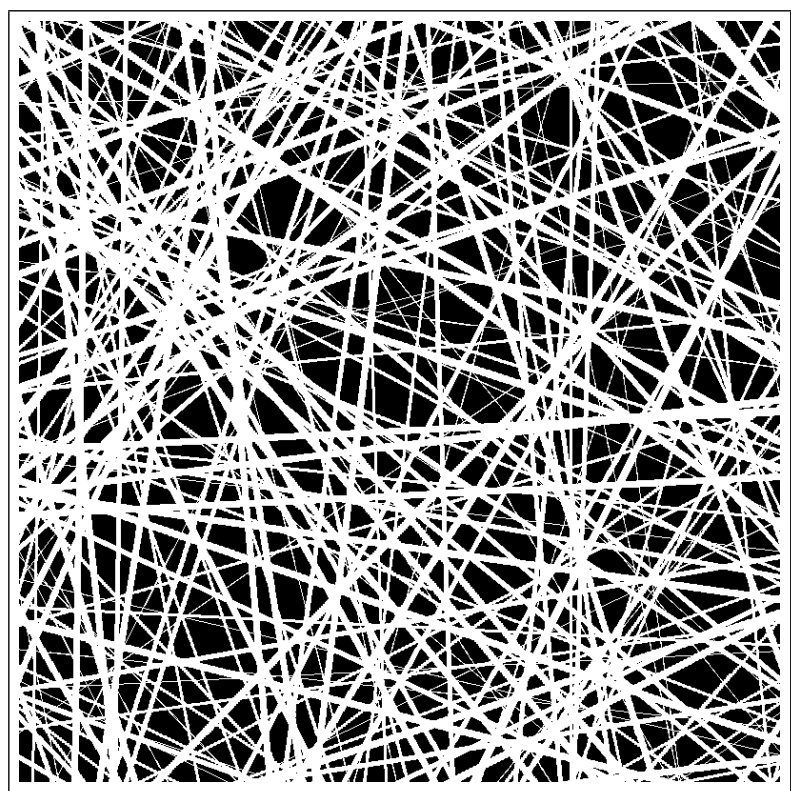

Figure 1: Simulation of a 2D Boolean model built on isotropic Poisson lines. 


\subsection{Random Fibers and Strata in 3D}

In $\mathbb{R}^{3}$, we can build a Boolean model on Poisson planes or on Poisson lines.

\subsubsection{Boolean model on Poisson planes}

A Boolean model built on Poisson planes generates a structure with strata. On isotropic Poisson planes, we have for a convex set $A^{\prime} \oplus \check{K}$ by application of equation (7):

$$
T(K)=1-\exp \left(-\theta \bar{M}\left(A^{\prime} \oplus \check{K}\right)\right)
$$

When $A^{\prime}$ and $K$ are convex sets, we have $\bar{M}\left(A^{\prime} \oplus \check{K}\right)=\bar{M}\left(A^{\prime}\right)+M(K)$. If $A^{\prime} \oplus \check{K}$ is not convex, $T(K)$ is expressed as a function of the length $l$ of the projection over the lines $D_{\omega}$ by $T(K)=1-\exp \left(-\theta \int_{2 \pi s t e r} \bar{l}\left(A^{\prime}(\omega) \oplus \check{K}(\omega)\right) d \omega\right)$. For instance if $A^{\prime}$ is a random sphere with a random radius $R$ (with expectation $\bar{R}$ ) and $K$ is a sphere with radius $r$, equation 10 becomes:

$$
\begin{aligned}
& T(r)=1-\exp (-4 \pi \theta(\bar{R}+r)) \\
& T(0)=P\{x \in A\}=1-\exp (-4 \pi \theta \bar{R})
\end{aligned}
$$

which can be used to estimate $\theta$ and $\bar{R}$, and to validate the model. Figure 1 can be interpreted as a 2D section of a 3D Boolean model built on Poisson hyperplanes. It was shown in (Jeulin 2001) that a two-components microstructure made of an infinite superposition of random sets made of dilated isotropic Poisson planes with a large separation of scales owns extremal physical effective properties (like electric conductivity, or elastic properties): when the highest conductivity is attributed to the dilated planes, the effective conductivity is the upper Hashin-Shtrikman bound, while it is equal to the lower Hashin-Shtrikman bound, when affecting the lower conductivity to the dilated planes.

\subsubsection{Boolean model on Poisson lines}

A Boolean model built on Poisson lines generates a fiber network, with possible overlaps of fibers. On isotropic Poisson lines, we have for a convex set $A^{\prime} \oplus \check{K}$

$$
T(K)=1-\exp \left(-\theta \frac{\pi}{2} \bar{S}\left(A^{\prime} \oplus \check{K}\right)\right)
$$

If $A^{\prime} \oplus \check{K}$ is not a convex set, $T(K)$ is expressed as a function of the area $A$ of the projection over the planes $\Pi_{\omega}$ by

$$
T(K)=1-\exp \left(-\theta \int_{2 \pi s t e r} \bar{A}\left(A^{\prime}(\omega) \oplus \check{K}(\omega)\right) d \omega\right)
$$


If $A^{\prime}$ is a random sphere with a random radius $R$ (with expectation $\bar{R}$ and second moment $E\left(R^{2}\right)$ ) and $K$ is a sphere with radius $r$, equation 11 becomes:

$$
\begin{aligned}
& T(r)=1-\exp \left(-\frac{\pi^{2}}{2} \theta\left(E\left(R^{2}\right)+2 r \bar{R}+r^{2}\right)\right) \\
& T(0)=P\{x \in A\}=1-\exp \left(-\frac{\pi^{2}}{2} \theta E\left(R^{2}\right)\right)
\end{aligned}
$$

which can be used to estimate $\theta, E\left(R^{2}\right)$ and $\bar{R}$, and to validate the model. A model of Poisson fibers parallel to a plane, and with a uniform distribution of orientations in the plane was used to model cellulosic fiber materials (Delisée et al. 2001). In (Schladitz et al. 2006), non isotropic dilated Poisson lines were used to model and to optimize the acoustic absorption of nonwoven materials.

\section{Fluctuations and RVE of the volume fraction}

When operating on bounded domains, like 2D or 3D images of a material, one can be concerned by estimating the fluctuations of spatial average values $\bar{Z}(V)$ of some random function $Z(x)$ over the domain $B$ with volume $V$. For instance if $Z(x)$ is the indicator function of a random set $A, \bar{Z}(V)$ is the area (in 2D) or the volume (in 3D) of the intersection $A \cap V$. If $Z(x)$ is some component of the strain field or of a stress field in an elastic medium, we can compute as well the average of these components over $V$, which are the standard way to define and to estimate the effective properties by homogenization (Jeulin 2001; Jeulin 2005; Kanit et al. 2003).

When working on images of a material or on realizations of a random medium, it is common to consider the representativity of the volume fraction or of the effective property estimated on a bounded domain of a microstructure. Practically, we need to estimate the size of a so-called "Representative Volume Element" RVE (Hersant and Jeulin 1976; Jeulin 2001; Jeulin 2005; Kanit et al. 2003). We address this problem by means of a probabilistic approach giving size-dependent intervals of confidence, and based on the size effect of the variance of the effective properties of simulations of random media.

\subsection{The integral range and scaling of the variance}

We consider fluctuations of average values over different realizations of a random medium inside the domain $B$ with the volume $V$. In Geostatistics (Matheron 1971), it is well known that for an ergodic stationary random function $Z(x)$, with mathematical expectation $E(Z)$, one can compute the variance $D_{Z}^{2}(V)$ of its average value $\bar{Z}(V)$ over the volume $V$ as a function 
of the central covariance function $\bar{C}(h)$ of $Z(x)$ by :

$$
D_{Z}^{2}(V)=\frac{1}{V^{2}} \int_{B} \int_{B} \bar{C}(x-y) d x d y,
$$

where

$$
\bar{C}(h)=E\{(Z(x)-E(Z))(Z(x+h)-E(Z))\}
$$

For a large specimen (with $V \gg A_{3}$ ), equation (13) can be expressed to the first order in $1 / V$ as a function of the integral range in the space $R^{3}, A_{3}$, by

$$
\begin{aligned}
D_{Z}^{2}(V) & =D_{Z}^{2} \frac{A_{3}}{V}, \\
\text { with } A_{3} & =\frac{1}{D_{Z}^{2}} \int_{R^{3}} \bar{C}(h) d h,
\end{aligned}
$$

where $D_{Z}^{2}$ is the point variance of $Z(x)$ (here estimated on simulations) and $A_{3}$ is the integral range of the random function $Z(x)$, defined when the integral in equations (13) and (15) is finite. When $Z(x)$ is the indicator function of the random set $A,(14)$ provides the variance of the local volume fraction (in 3D) as a function of the point variance $D_{Z}^{2}=p(1-p), p$ being the probability for a point $x$ to belong to the random set $A$. When working in $2 \mathrm{D}$, as was done to solve sampling problems in image analysis (Hersant and Jeulin 1976), the volume $V$ is replaced by the surface area, and the integral range becomes $A_{2}$ after integrating the covariance in the 2D space $R^{2}$ in equation 15. The asymptotic scaling law (14) is valid for an additive variable $Z$ over the region of interest $B$. To estimate the effective elasticity or permittivity tensors from simulations, we have to compute the spatial average stress $\langle\sigma\rangle$ and strain $\langle\varepsilon\rangle$ (elastic case) or electric displacement $\langle D\rangle$ and electrical field $\langle E\rangle$. For the applied boundary conditions, the local modulus is obtained from the estimations of a scalar, namely the average in the domain $B$ of the stress, strain, electric displacement, or electric field. Therefore the variance of the local effective property follows the equation (14) when the integral range $A_{3}$ of the relevant field is known. Since the theoretical covariance of the fields ( $\sigma$ or $\varepsilon$ ) is not available, the integral range can be estimated according to the procedure proposed by Matheron (1989) for any random function : working with realizations of $Z(x)$ on domains $B$ with an increasing volume $V$ (or in the present case considering subdomains of large simulations, with a wide range of sizes), the parameter $A_{3}$ is estimated by fitting the obtained variance according to the expression (14).

Some typical microstructures with long range correlations, like dilated Poisson hyperplanes mentioned in section 3.2.1 or like dilated Poisson lines in 3D have an infinite integral range (Jeulin 1991; Jeulin 1991), so that the computation of the variance $D_{Z}^{2}(V)$ of equation (14) cannot be used anymore. In this situation, a scaling law by a power $\gamma<1$ was suggested (Lantuejoul 1991), and used in various applications where a coefficient close 
to 1 was empirically estimated (Cailletaud et al. 1994; Kanit et al. 2003). With this scaling law, the variance becomes

$$
D_{Z}^{2}(V)=D_{Z}^{2}\left(\frac{A_{3}}{V}\right)^{\gamma},
$$

where the volume $A_{3}$ is no more the integral of the central covariance function $\bar{C}(h)$, but is still homogeneous to a microstructural volume. We will show in section 5, that such theoretical scaling power laws appear in the case of Boolean models built on the linear Poisson varieties.

\subsection{Practical determination of the size of the RVE}

The size of a RVE can be defined for a physical property $Z$, a contrast, and a given precision in the estimation of the effective properties depending on the number $n$ of realizations that are available. By means of a standard statistical approach, the absolute error $\epsilon_{a b s}$ and the relative error $\epsilon_{\text {rela }}$ on the mean value obtained with $n$ independent realizations of volume $V$ are deduced from the $95 \%$ interval of confidence by:

$$
\epsilon_{a b s}=\frac{2 D_{Z}(V)}{\sqrt{n}} ; \epsilon_{\text {rela }}=\frac{\epsilon_{a b s}}{Z}=\frac{2 D_{Z}(V)}{Z \sqrt{n}} .
$$

The size of the RVE can now be defined as the volume for which for instance $n=1$ realization (as a result of an ergodicity assumption on the microstructure) is necessary to estimate the mean property $Z$ with a relative error (for instance $\epsilon_{\text {rela }}=1 \%$ ), provided we know the variance $D_{Z}^{2}(V)$ from the asymptotic scaling law (14) or (16). Alternatively, we can decide to operate on smaller volumes (provided no bias is introduced by the boundary conditions), and consider $n$ realizations to obtain the same relative error. This methodology was applied, among others, to the elastic properties and thermal conductivity of a Voronoï mosaic (Kanit et al. 2003), of materials from food industry (Kanit et al. 2006), or of Boolean models of spheres (Willot and Jeulin 2009).

\section{Scaling of the variance of the Boolean random varieties}

\subsection{Boolean model on Poisson varieties in $\mathbb{R}^{n}$}

We consider a convex domain $K$ in $\mathbb{R}^{n}$, with Lebesgue measure $\mu_{n}(K)$. Deriving the asymptotic expression of the local fraction (with average $p$ ) from the covariance (8) in expression (13) is not an easy task. The scaling law of the variance (16) can be directly obtained for the Boolean model built on isotropic Poisson varieties $V_{k}$ from the properties of the Poisson point process. We have the following result. 
Proposition 6 In $\mathbb{R}^{n}$, the variance $D_{Z}^{2}(K)$ of the local fraction $Z=\frac{\mu_{n}(A \cap K)}{\mu_{n}(K)}$ of a Boolean model built on isotropic Poisson varieties of dimension $k$ ( $k=$ $0,1, \ldots, n-1) V_{k}$, is asymptotically expressed, for homothetic convex sets $K$ with $\mu_{n}(K) \rightarrow \infty$, by

$$
D_{Z}^{2}(K)=p(1-p)\left(\frac{A_{k}(K)}{\mu_{n}(K)}\right)^{\frac{n-k}{n}}
$$

the scaling exponent being $\gamma=\frac{n-k}{n}$. The coefficient $A_{k}(K)$ depends on the shape of $K$, but not on its size. As particular cases, Poisson points $(k=0)$ give the standard Boolean model with a finite integral range and $\gamma=1$, Poisson lines $(k=1)$ generate Poisson fibers with $\gamma=\frac{n-1}{n}$, and Poisson hyperplanes $(k=n-1)$ provide Poisson strata with $\gamma=\frac{1}{n}$.

Proof. Consider isotropic varieties $V_{k}$ with dimension $k$ and intensity $\theta_{k}$. From proposition 3, the number of varieties hit by $K$ follows a Poisson distribution with average and variance proportional to $\theta_{k} W_{k}(K)$. To express the scaling law of the variance $D_{Z}^{2}(K)$, we consider the limiting case of a low intensity $\theta_{k}$ in expression (5) for large $K$ as compared to the primary grain $A^{\prime}\left(\bar{\mu}_{n}\left(A^{\prime}\right)<<\mu_{n}(K)\right)$, so that to first order $W_{k}(K) \approx W_{k}\left(A^{\prime} \oplus \check{K}\right)$. For a given realization of $V_{k}$, the measure $\mu_{n}(A \cap K)$ is proportional to $\mu_{k}\left(V_{k} \cap K\right)$. As a result of the ergodicity of the random varieties $V_{k}$, for large $K, \mu_{k}\left(V_{k} \cap K\right)$ converges towards the mathematical expectation of the measure of random sections of $K, E\left\{\mu_{k}\left(V_{k} \cap K\right)\right\}$. Its value can be deduced from the Crofton formula given in (Matheron 1975), p. 82, or from (Klain and Rota 1997) p. 129. We get:

$$
E\left\{\mu_{k}\left(V_{k} \cap K\right)\right\}=\frac{\mu_{n}(K)}{\mu_{n-k}(K)} \frac{n !}{k !(n-k) !}
$$

Making use of (5), the local volume fraction of $A, \frac{\mu_{n}(A \cap K)}{\mu_{n}(K)}$ has an expectation proportional to $\theta_{k} W_{k}(K) \frac{1}{\mu_{n-k}(K)} \approx \theta_{k}$ and a variance proportional to

$$
\theta_{k} W_{k}(K) \frac{1}{\left(\mu_{n-k}(K)\right)^{2}} \approx \frac{1}{\mu_{n-k}(K)} \approx \frac{1}{\mu_{n}(K)^{\frac{n-k}{n}}}
$$

It turns out that the most penalizing situation with respect to the scaling of the variance is the case of Poisson strata, with a very slow decrease of the variance with the volume of the sample $K$, with $\gamma=\frac{1}{n}$.

It is difficult to give equivalent results for general non-isotropic models. Instead, we will give below some specific examples useful for applications in $2 D$ and in $3 D$. 


\subsection{Random fibers in $2 \mathrm{D}$}

- For isotropic Boolean fibers in 2D, the scaling exponent is $\gamma=\frac{1}{2}$. Note that in that case the Poisson varieties are Poisson lines, which are at the same time lines and hyperplanes in $\mathbb{R}^{2}$.

- An instructive and extreme anisotropic case is obtained for parallel Poisson lines, with the intensity $\theta_{1}(d \alpha)=\theta_{1} \delta\left(\alpha_{0}\right) d \alpha, \alpha_{0}$ being the orientation of the lines. For a sample $K$ made of a rectangle with an edge $L$ orthogonal to $\alpha_{0}$, and an edge $l$ parallel to $\alpha_{0}$. The number of lines hitting $K$ is a Poisson variable with average and variance $\theta_{1} L$. The average of the local area fraction of $A$ is proportional to $\theta_{1} L \frac{l}{L l}=$ $\theta_{1} L \frac{1}{L}$ and its variance is proportional to $\theta_{1} L \frac{1}{L^{2}}=\frac{\theta_{1}}{L}$. The length $l$ plays no role in the variance, which is inversely proportional to $L$. In that case, $1 \mathrm{D}$ samples orthogonal to $\alpha_{0}$ produce the same scaling of the variance. This can be explained by the fact that the $1 \mathrm{D}$ sections of the model orthogonal to $\alpha_{0}$ are a standard one-dimensional Boolean model with a finite integral range, showing a standard scaling of the variance in $1 \mathrm{D}$.

\subsection{Random fibers in 3D}

- For isotropic Boolean fibers in 3D, the scaling exponent is $\gamma=\frac{2}{3}$. This exponent was recovered for the volume fraction from numerical simulations (Dirrenberger et al. 2014) for $V_{V} \simeq 0.16$. In the case of fibers with a finite length, the expected scaling coefficient is $\gamma=1$. However an intermediary situation will occur in experiments, and an apparent scaling coefficient $\frac{2}{3} \leqslant \gamma \leqslant 1$, depending on the size of the specimen can be recovered ( $\gamma \simeq \frac{2}{3}$ for small specimens, and $\gamma \simeq 1$ for large samples). Simulations of various random networks of finite fibers having a length of the order of the size of the samples, and with various radius distributions (Table 3 in Altendorf et al. 2014), gave apparent $\gamma \simeq 0.66-0.87$ instead of the true $\gamma=1$, but mostly around 0.8 , for the volume fraction, with $V_{V} \simeq 0.15$. Simulations of the elastic properties and of the conductivity of 3D random fiber networks by finite elements (Dirrenberger et al. 2014) or by FFT (Altendorf et al. 2014) provide an empirical scaling law of the variance close to the theoretical one obtained for the volume fraction. This is expected, as a result of a high correlation between the elastic or thermal fields and the indicator function of the random set $A$.

- An extreme case of anisotropy is given for fibers parallel to a direction $\alpha_{0}$, providing a standard 2D Boolean model in planes orthogonal to $\alpha_{0}$. Consider for $K$ a parallelepiped with a face orthogonal to the direction $\alpha_{0}$ (with area $S$ ), and an edge parallel to the direction 
$\alpha_{0}$ (with length $l$ ). The number of Poisson lines hitting $K$ is a Poisson variable with parameter $\theta_{1} S$, and 2D sections of $A$ generate a standard 2D Boolean model. The average of the local volume fraction of $A$ is proportional to $\theta_{1} S \frac{l}{S l}=\theta_{1} S \frac{1}{S}$ and its variance is proportional to $\theta_{1} S \frac{1}{S^{2}}=\frac{\theta_{1}}{S}$. As in $1 \mathrm{D}$, the length $l$ plays no role in the variance, which is inversely proportional to $S$. The same scaling is obtained for $2 \mathrm{D}$ sections in planes orthogonal to the direction $\alpha_{0}$. This was observed on a silica fibers composite, for the fluctuations of the area fraction (with $A_{A}=0.4$ ) and of the elastic and thermal fields, calculated by finite elements on polished sections of the composite (Oumarou et al. 2011). Planar sections parallel to $\alpha_{0}$ generate a $2 \mathrm{D}$ model of Boolean fibers. The variance is proportional to $\frac{\theta_{1}}{L}, L$ being the length of the edge orthogonal to $\alpha_{0}$, the edge parallel to the fibers playing no roles. In this situation, one dimensional sections orthogonal to $\alpha_{0}$, give the same scaling law for the variance, that is decreasing much slower than for transverse sections or for the isotropic model.

- It is possible to model a random woven composite by a network of random fibers with a set of orientations, for instance two or three orthogonal orientations $\alpha_{1}, \alpha_{2}, \alpha_{3}$, and corresponding intensities $\theta_{1}$, $\theta_{2}, \theta_{3}$. Consider for $K$ a parallelepiped with a face orthogonal to the direction $\alpha_{1}$ (with area $S_{1}$ ), and faces orthogonal to direction $\alpha_{2}$ (with area $S_{2}$ ) and to direction $\alpha_{3}$ (with area $S_{3}$ ). The lengths of the edges in direction $\alpha_{i}$ are $L_{i}(i=1,2,3)$, so that $S_{1}=L_{2} L_{3}, S_{2}=L_{1} L_{3}$ and $S_{3}=L_{2} L_{1}$. In the case of two orthogonal directions $\alpha_{1}, \alpha_{2}$, the variance scales as $\frac{\theta_{1}}{S_{1}}+\frac{\theta_{2}}{S_{2}}$, while for three orthogonal orientations, it scales as $\frac{\theta_{1}}{S_{1}}+\frac{\theta_{2}}{S_{2}}+\frac{\theta_{3}}{S_{3}}$. For a cube, an overall scaling in $\frac{\theta}{S}$ is recovered with $\theta=\theta_{1}+\theta_{2}+\theta_{3}$, which still gives a scaling exponent $\gamma=\frac{2}{3}$. The microstructure can also be sampled by planar probes $K$. For two directions of fibers $\alpha_{1}, \alpha_{2}$ and cuts orthogonal to direction $\alpha_{1}$, the variance scales as $\frac{\theta_{1}}{S_{1}}+\frac{\theta_{2}}{L_{2}}$. For cuts parallel to the plane defined by orientations $\alpha_{1}, \alpha_{2}$ the variance scales as $\frac{\theta_{1}}{L_{1}}+\frac{\theta_{2}}{L_{2}}$, which is the most penalizing situations. These results extend to three orthogonal orientations, with a variance scaling as $\frac{\theta_{1}}{S_{1}}+\frac{\theta_{2}}{L_{2}}+\frac{\theta_{3}}{L_{3}}$ for cuts orthogonal to direction $\alpha_{1}$, which are therefore parallel to the plane defined by directions $\alpha_{2}, \alpha_{3}$.

- Some fibrous materials, like cellulosic fibrous media (Delisée et al. 2001), are isotropic transverse: fibers are parallel to a reference plane, orthogonal to some direction $\alpha_{3}$, with a uniform distribution of orientations in this plane. The number of Poisson line hit by $K$ follows a Poisson distribution with parameter $\theta\left(L_{1}+L_{2}\right) L_{3}$, and the variance of the local volume fraction scales as $\frac{\theta}{\left(L_{1}+L_{2}\right) L_{3}}$. Planar sections with area $S$ of this model orthogonal to the direction $\alpha_{3}$ generate a $2 \mathrm{D}$ 
isotropic Boolean model of fibers with the scaling exponent $\gamma=\frac{1}{2}$ for $\frac{1}{S}$, as in 5.2 .

- Projections of 3D Boolean fibers networks on a plane generate various standard models with the corresponding scaling laws. Any projection of the isotropic network, or a projection of transverse isotropic fibers in a plane parallel to fibers (Delisée et al. 2001) are 2D isotropic Boolean fibers with he scaling exponent $\gamma=\frac{1}{2}$ for $\frac{1}{S}$ as in 5.2. The projection of parallel fibers in a plane parallel to fibers generates $2 \mathrm{D}$ Boolean parallel fibers, with a variance scaling as $\frac{1}{L}, L$ being the length of the edge of the section orthogonal to fibers. The projection of parallel fibers in a plane orthogonal to fibers generates standard 2D Boolean model with a scaling of the variance in $\frac{1}{S}$. This models appear by observation of thick slides of fibrous networks, as obtained by optical confocal microscopy (Delisée et al. 2001), or from transmission electron microscopy.

\subsection{Random strata in 3D}

- For isotropic Boolean strata in 3D, the scaling exponent is $\gamma=\frac{1}{3}$. This decrease of the variance with size is much slower than the case of a finite integral range. When considering random media with a nonlinear behavior, like for instance a viscoplastic material, a strong localization of strains resulting in shear bands is expected. This generates long range correlations of the fields, that might be modeled by Boolean strata in 3D, and scaling laws similar to the dilated Poisson hyperplanes (with a scaling exponent close to $\frac{1}{3}$ ) might be recovered, so that a slow convergence towards the effective properties should be observed on numerical simulations with increasing sizes. This point remains to be investigated by numerical experiments.

- A layered medium, generated by parallel hyperplanes, orthogonal to a reference direction $\alpha_{0}$. corresponds to an extremal anisotropy. Consider for $K$ a parallelepiped with a face orthogonal to the direction $\alpha_{0}$ (with area $S$ ). The length of the edge parallel to $\alpha_{0}$ is $L$. The number of planes hit by $L$ is a Poisson variable with parameter $\theta L$. The local volume fraction has an expectation equal to $\theta L \frac{S}{V}=\theta$ and its variance is proportional to $\theta L\left(\frac{S}{V}\right)^{2}=\frac{\theta}{L}$. For this situation, there is no effect of the surface $S$, and plane sections parallel to the direction $\alpha_{0}$ gives the same scaling of the variance.

- Another instructive case is obtained by isotropic hyperplanes parallel to a given direction $\alpha_{0}$. Consider for $K$ a parallelepiped with a face orthogonal to the direction $\alpha_{0}$ (with perimeter $\mathcal{L}$ and area $S$ ). The number of Poisson planes hit by $K$ follows a Poisson distribution with 
parameter $\theta \mathcal{L}$. If the length of $K$ in the direction parallel to $\alpha_{0}$ is $L$, the local volume fraction has an expectation equal to $\theta \mathcal{L} \frac{\mathcal{L} L}{V}=\theta$, and its variance is proportional to $\theta \mathcal{L}\left(\frac{\mathcal{L} L}{V}\right)^{2} \approx \frac{\theta}{S^{1 / 2}} \approx \frac{\theta}{V^{1 / 3}}$. Planar sections (or equivalently projections) orthogonal to $\alpha_{0}$ generate isotropic $2 \mathrm{D}$ Boolean fibers, with a variance scaling in $\frac{\theta}{S^{1 / 2}}$. Vertical planar sections, parallel to $\alpha_{0}$ (with a horizontal edge $L$ ) generate parallel 2D Boolean fibers, with a variance scaling in $\frac{\theta}{L}$, the size of the vertical edge playing no role in the variance.

- As for fibers, It is possible to model a random woven composite by a network of random strata with a set of orientations, for instance two or three orthogonal orientations $\alpha_{1}, \alpha_{2}, \alpha_{3}$. Consider for $K$ a parallelepiped with edges parallel to these orientations, and lengths $L_{1}, L_{2}$, and $L_{3}$. The variance scales as $\frac{\theta_{1}}{L_{1}}+\frac{\theta_{2}}{L_{2}}+\frac{\theta_{3}}{L_{3}}$. When $K$ is a cube with edge $L$, the variance scales as $\frac{\theta_{1}+\theta_{2}+\theta_{3}}{L}$.

\section{Two steps Boolean varieties}

It is possible to generate further Boolean models by iteration of Poisson varieties. For instance in $\mathbb{R}^{2}$, we first consider Poisson line, and in a second step Poisson points on every lines. These points are germs to locate primary grains $A^{\prime}$ to generate a Boolean model. Compared to the standard Boolean model, this one shows alignments of grains. Similarly in $\mathbb{R}^{3}$ we can start from Poisson planes $V_{2 \alpha}$ and use Poisson lines $V_{1 \beta}$ in every plane to generate a Boolean model with fibers. In contrast with Poisson fibers in $\mathbb{R}^{3}$, this model generates a random set with some coplanar fibers. Such long range random sets could mimic specific microstructures with an order in a lower dimension subspace of $\mathbb{R}^{n}$, such as preferred germination of objects on specific planes or lines.

These models are based on doubly stochastic Poisson random variables for which the Choquet capacity and scaling laws of the variance can be obtained.

Definition 7 Two steps random varieties are defined as follows: starting from Poisson linear varieties $V_{k}$ of dimension $k$ and with intensity $\theta_{k}(d \omega)$ in $\mathbb{R}^{n}$, Poisson linear varieties $V_{k^{\prime} \beta}$ with dimension $0 \leq k^{\prime}<k$ and with intensity $\theta_{k^{\prime}}(d \omega)$ are implanted on each $V_{k \alpha}$. Then each $V_{k^{\prime} \beta}$ is dilated by independent realizations of a random compact primary grain $A^{\prime} \subset \mathbb{R}^{n}$ to generate the Boolean RACS A:

$$
A=\cup_{\beta} V_{k^{\prime} \beta} \oplus A^{\prime}
$$


Remark 8 By construction, when $k^{\prime}=0$ the varieties $V_{k^{\prime}} \beta$ are a particular case of a Cox process driven by the random set $V_{k}$, and the derived random set $A$ is a Cox Boolean model (Jeulin 2012).

In what follows the purpose is restricted to the stationary and isotropic case, with the two intensities $\theta_{k}$ and $\theta_{k^{\prime}}$.

Theorem 9 The number $N(K)$ of varieties of dimension $k^{\prime}<k$ hit by the compact set $K$ is a random variable with generating function

$$
G_{k^{\prime}}(s, K)=E\left\{s^{N(K)}\right\}=\exp \left[\theta_{k} a_{k} W_{k}(K)\left[\varphi_{k^{\prime}}\left(\theta_{k^{\prime}} a_{k^{\prime}}(1-s), K\right)-1\right]\right]
$$

where $a_{k^{\prime}}=\frac{b_{n-k^{\prime}} b_{k^{\prime}+1}}{b_{n}} \frac{k^{\prime}+1}{2}$ and $\varphi_{k^{\prime}}(\lambda, K)$ is the Laplace transform of the random variable $W_{k^{\prime}}\left(K \cap V_{k \alpha}\right), W_{k^{\prime}}$ being the Minkowski functional homogeneous with degree $k-k^{\prime}$ in $\mathbb{R}^{k}$ :

$$
\varphi_{k^{\prime}}(\lambda, K)=E\left\{\exp \left[-\lambda W_{k^{\prime}}\left(K \cap V_{k}\right)\right]\right\}
$$

the mathematical expectation being taken over the realizations $V_{k \alpha}$. As a consequence, the Choquet capacity of the Boolean RACS A built on the Poisson linear varieties $V_{k^{\prime}}$ using a deterministic primary grain $A^{\prime}$ is derived from $G_{k^{\prime}}\left(0, A^{\prime} \oplus \check{K}\right), E\{\}$ being the expectation with respect to the random variety $V_{k \alpha}$ :

$$
\begin{aligned}
& 1-T(K) \\
& =\exp \left[-\theta_{k} a_{k} W_{k}\left(A^{\prime} \oplus \check{K}\right)\left[1-E\left\{\exp \left[-\theta_{k^{\prime}} a_{k^{\prime}} W_{k^{\prime}}\left(A^{\prime} \oplus \check{K} \cap V_{k}\right)\right]\right\}\right]\right]
\end{aligned}
$$

Proof. The random number $N_{k}$ of varieties $V_{k \alpha}$ hit by $K$ is a Poisson variable with expectation $\theta_{k} a_{k} W_{k}(K)$. On each $V_{k \alpha}$ are generated $N_{k^{\prime}}$ varieties $V_{k^{\prime} \beta}, N_{k^{\prime}}$ being a Poisson variable with expectation $\theta_{k^{\prime}} a_{k^{\prime}} W_{k^{\prime}}\left(K \cap V_{k}\right)$. For a random section $K \cap V_{k \alpha}$, the generating function of $N_{k^{\prime}}$ is

$$
\Gamma(s)=\exp \left[-\theta_{k^{\prime}} a_{k^{\prime}} W_{k^{\prime}}\left(K \cap V_{k \alpha}\right)(1-s)\right]
$$

Taking the expectation of (22) with respect to $W_{k^{\prime}}\left(K \cap V_{k \alpha}\right)$ and then of $\Gamma(s)^{N_{k}}$ gives (19).

The Choquet capacity requires the use of the Laplace transform $\varphi_{k^{\prime}}\left(\lambda, A^{\prime} \oplus\right.$ $\check{K})$. It is not easy to expressed it in a closed form for specific compact sets $K$ and $A^{\prime}$. However the required distribution functions and their Laplace transforms can be estimated by simulation of the random variables obtained from random variables $W_{k^{\prime}}\left(A^{\prime} \oplus \check{K} \cap V_{k \alpha}\right)$ obtained from random sections.

As in section 5 it is possible to work out asymptotic scaling exponents of the local volume fraction of $A, \frac{\mu_{n}(A \cap K)}{\mu_{n}(K)}$, starting from the variance of the random number $N(K)$. Using the generating function $G_{k^{\prime}}(s, K)$ given in equation (19), we have

$$
D^{2}(N(K))=G_{k^{\prime}}^{\prime \prime}(1, K)+G_{k^{\prime}}^{\prime}(1, K)\left(1-G_{k^{\prime}}^{\prime}(1, K)\right)
$$




$$
\begin{gathered}
G_{k^{\prime}}^{\prime}(s, K)=-\theta_{k} a_{k} W_{k}(K) \theta_{k^{\prime}} a_{k^{\prime}}\left[\varphi_{k^{\prime}}^{\prime}\left(\theta_{k^{\prime}} a_{k^{\prime}}(1-s), K\right)\right] G_{k^{\prime}}(s, K) \\
\begin{array}{r}
G_{k^{\prime}}^{\prime}(1, K)=E\{N(K)\} \\
=-\theta_{k} a_{k} W_{k}(K) \theta_{k^{\prime}} a_{k^{\prime}}\left[\varphi_{k^{\prime}}^{\prime}(0, K)\right] \\
=\theta_{k} a_{k} W_{k}(K) \theta_{k^{\prime}} a_{k^{\prime}} E\left\{W_{k^{\prime}}\left(K \cap V_{k}\right)\right\}
\end{array} \\
\begin{array}{r}
G_{k^{\prime}}^{\prime \prime}(s, K) \quad \\
=\theta_{k} a_{k} W_{k}(K) \theta_{k^{\prime}} a_{k^{\prime}}\left[\varphi_{k^{\prime}}^{\prime \prime}\left(\theta_{k^{\prime}} a_{k^{\prime}}(1-s), K\right)\right] G_{k^{\prime}}(s, K) \\
-\theta_{k} a_{k} W_{k}(K) \theta_{k^{\prime}} a_{k^{\prime}}\left[\varphi_{k^{\prime}}^{\prime}\left(\theta_{k^{\prime}} a_{k^{\prime}}(1-s), K\right)\right] G_{k^{\prime}}^{\prime}(s, K)
\end{array}
\end{gathered}
$$

and

$$
\begin{aligned}
& G_{k^{\prime}}^{\prime \prime}(1, K) \\
& =\theta_{k} a_{k} W_{k}(K) \theta_{k^{\prime}} a_{k^{\prime}}\left[\varphi_{k^{\prime}}^{\prime \prime}(0, K)\right]+\left[G_{k^{\prime}}^{\prime}(1, K)\right]^{2} \\
& =\theta_{k} a_{k} W_{k}(K) \theta_{k^{\prime}} a_{k^{\prime}} E\left\{\left(W_{k^{\prime}}\left(K \cap V_{k}\right)\right)^{2}\right\}+(E\{N(K)\})^{2}
\end{aligned}
$$

so that

$$
\begin{aligned}
& D^{2}(N(K)) \\
& =\theta_{k} a_{k} W_{k}(K) \theta_{k^{\prime}} a_{k^{\prime}}\left[\varphi_{k^{\prime}}^{\prime \prime}(0, K)\right]+\theta_{k} a_{k} W_{k}(K) \theta_{k^{\prime}} a_{k^{\prime}} E\left\{W_{k^{\prime}}\left(K \cap V_{k}\right)\right\} \\
& =\theta_{k} a_{k} W_{k}(K) \theta_{k^{\prime}} a_{k^{\prime}}\left[E\left\{\left(W_{k^{\prime}}\left(K \cap V_{k}\right)\right)^{2}\right\}+E\left\{W_{k^{\prime}}\left(K \cap V_{k}\right)\right\}\right]
\end{aligned}
$$

As in section 5, the local volume fraction of the random set $A$ in $K$ is proportional to $\mu_{k^{\prime}}\left(K \cap V_{k^{\prime}}\right)$. As a result of the ergodicity of the random varieties $V_{k}$, for large $K, \mu_{k^{\prime}}\left(V_{k^{\prime}} \cap K\right)$ converges towards its mathematical expectation. Applying the Crofton formula in two steps we get:

$$
E\left\{\mu_{k^{\prime}}\left(V_{k^{\prime}} \cap K\right) \mid V_{k}\right\}=\frac{\mu_{k}\left(K \cap V_{k}\right)}{\mu_{k-k^{\prime}}(K)} \frac{k !}{k^{\prime} !\left(k-k^{\prime}\right) !}
$$

and

$$
\begin{aligned}
E\left\{\mu_{k^{\prime}}\left(V_{k^{\prime}} \cap K\right)\right\} & =\frac{E\left\{\mu_{k}\left(K \cap V_{k}\right)\right\}}{\mu_{k-k^{\prime}}(K)} \frac{k !}{k^{\prime} !\left(k-k^{\prime}\right) !} \\
& =\frac{\mu_{n}(K)}{\mu_{n-k}(K)} \frac{n !}{k !(n-k) !} \frac{1}{\mu_{k-k^{\prime}}(K)} \frac{k !}{k^{\prime} !\left(k-k^{\prime}\right) !}
\end{aligned}
$$


The local volume fraction of $A, \frac{\mu_{n}(A \cap K)}{\mu_{n}(K)}$ has a variance proportional to

$$
\begin{aligned}
& \frac{1}{\left(\mu_{n-k}(K) \mu_{k-k^{\prime}}(K)\right)^{2}} D^{2}(N(K)) \\
& \approx \frac{1}{\left(\mu_{n-k}(K) \mu_{k-k^{\prime}}(K)\right)^{2}} W_{k}(K) E\left\{\left(W_{k^{\prime}}\left(K \cap V_{k}\right)\right)^{2}\right\} \\
& \approx \frac{\mu_{n-k}(K)\left(\mu_{k-k^{\prime}}(K)\right)^{2}}{\left(\mu_{n-k}(K) \mu_{k-k^{\prime}}(K)\right)^{2}} \approx \frac{1}{\mu_{n-k}(K)} \approx \frac{1}{\mu_{n}(K) \frac{n-k}{n}}
\end{aligned}
$$

so that the scaling law of the variance is the same as in (18). Therefore the variance of the volume fraction of iterated varieties is dominated by the effect of the varieties of the first iteration, namely $V_{k}$. For instance in $\mathbb{R}^{2}$ a Boolean model built on Poisson lines or on Poisson points generated on Poisson lines have the same scaling with the exponent $\gamma=\frac{1}{2}$. In $\mathbb{R}^{3} \mathrm{a}$ Boolean model built on Poisson lines or on Poisson points in Poisson planes have the same scaling with the exponent $\gamma=\frac{1}{3}$, while for a Boolean model built on Poisson fibers or on Poisson points located on Poisson lines we have $\gamma=\frac{2}{3}$.

\section{Conclusion}

Boolean random varieties generate random media with infinite range correlations. As a consequence, non standard scaling laws of the variance of the local volume fraction with the volume of domains $K$ are predicted. These laws are out of reach of a standard statistical approach. We have theoretically shown that on a large scale, the variance of the local volume fraction decreases with power laws of the volume of $K$. The exponent $\gamma$ is equal to $\frac{2}{3}$ for Boolean fibers in 3D, and $\frac{1}{3}$ for Boolean strata in 3D. When working in $2 \mathrm{D}$, the scaling exponent of Boolean fibers is equal to $\frac{1}{2}$. Therefore the decrease of the variance with the scale is much slower for these models as compared to situations with a finite integral range, like the standard Boolean model built on a Poisson point process with compact primary grains, and larger RVE are expected for the estimation of the volume fraction. From numerical simulations to predict the effective properties of such random media, it turns out that these laws seem to hold, as already empirically observed for the conductivity or for the elastic properties of random fibers models. We can forecast the development of long range correlations when there is a strong localization of strains resulting in shear bands for a non linear mechanical behavior. For these situations a power law scaling with $\gamma \simeq \frac{1}{3}$ is suspected to occur, but this has to be checked on data.

Similar results are obtained for iterated Poisson varieties, the first step of the iteration imposing its power law behavior for the scaling of the variance.

The obtained scaling laws in various cases, including anisotropic orientations of the Boolean fibers or strata, and the iterated Poisson varieties, 
can help to design optimal sampling schemes with respect to minimizing the variance of estimation.

\section{References}

[1] Altendorf H, Jeulin D, Willot F (2014). Influence of the fiber geometry on the macroscopic elastic and thermal properties. International Journal of Solids and Structures 51(23): 3807-3822

[2] Cailletaud G, Jeulin D, Rolland Ph (1994) Size effect on elastic properties of random composites, Engineering computations 11 (2): 99-110

[3] Delisée Ch, Jeulin D, Michaud F (2001) Caractérisation morphologique et porosité en 3D de matériaux fibreux cellulosiques, C.R. Académie des Sciences de Paris, t. 329, Série II b, 179-185

[4] Dirrenberger J, Forest S, Jeulin D (2014) Towards gigantic RVE sizes for 3D stochastic fibrous networks. International Journal of Solids and Structures 51(2), 359-376

[5] Hersant T, Jeulin D (1976) L'échantillonnage dans les analyses quantitatives d'images. Exemples d'application aux mesures des teneurs de phases dans les agglomérés et des inclusions dans les aciers, Mémoires et Etudes Scientifiques de la Revue de Métallurgie 73: 503.

[6] Jeulin D (1991) Modèles Morphologiques de Structures Aléatoires et de Changement d'Echelle. Thèse de Doctorat d'Etat ès Sciences Physiques, Université de Caen, 25 Avril 1991

[7] Jeulin D (1991) Modèles de Fonctions Aléatoires multivariables. Sci. Terre 30: 225-256

[8] Jeulin D (2001) Random Structure Models for Homogenization and Fracture Statistics, in: Mechanics of Random and Multiscale Microstructures, Jeulin D and Ostoja-Starzewski M (eds), CISM Lecture Notes $N^{\circ} 430$, Springer Verlag, 33-91

[9] Jeulin D (2005) Random Structures in Physics, in: Space, Structure and Randomness, Contributions in Honor of Georges Matheron in the Fields of Geostatistics, Random Sets, and Mathematical Morphology; Series: Lecture Notes in Statistics, Vol. 183, Bilodeau M, Meyer F, Schmitt M (eds.), XIV, Springer-Verlag, 183-222

[10] Jeulin D (2012) Morphology and effective properties of multi-scale random sets: A review, C. R. Mecanique 340: 219-229 
[11] Kanit T, Forest S, Galliet I, Mounoury V, Jeulin D (2003) Determination of the size of the representative volume element for random composites: statistical and numerical approach, International Journal of solids and structures 40: 3647-3679

[12] Kanit T, N'Guyen F, Forest S, Jeulin D, Reed M, Singleton S (2006) Apparent and effective physical properties of heterogeneous materials: Representativity of samples of two materials from food industry, Comput. Methods Appl. Mech. Eng. 195: 3960-3982

[13] Klain D A, Rota G C (1997) Introduction to Geometric Probability, Cambridge University Press, Cambridge

[14] Lantuejoul Ch (1991) Ergodicity and integral range, Journal of Microscopy 161: 387-403

[15] Matheron G (1971) The theory of regionalized variables and its applications, Paris School of Mines publications

[16] Matheron G (1975) Random sets and Integral Geometry, J. Wiley, N.Y

[17] Matheron G (1989) Estimating and Choosing, Springer Verlag, Berlin

[18] Oumarou M, Jeulin D, Renard J (2011) Etude statistique multi-échelle du comportement élastique et thermique d'un composite thermoplastique, Revue des composites et des matériaux avancés 21(2): 221-254

[19] Schladitz K, Peters S, Reinel-Bitzer D, Wiegmann A, Ohser J (2006) Design of acoustic trim based on geometric modeling and flow simulation for non-woven, Computational Materials Science 38: 56-66

[20] Willot F, Jeulin D (2009) Elastic behavior of composites containing Boolean random sets of inhomogeneities, International Journal of Engineering Sciences 47: 313-324 